\title{
BANDWIDTH ANALYSIS OF THIRD-HARMONIC GENERATION IN OPTICAL THIN FILMS
}

\author{
Gia-Wei Chern, Tzu-Min Liu, Shi-Wei Chu, Chi-Kuang Sun \\ Graduate Institute of Electro-Optical Engineering, National Taiwan University, \\ Taipei 10617, Taiwan \\ Phone: 886-2-23635251x319, Fax: 886-2-23677467, E-Mail: weichern@cc.ee.ntu.edu.tw
}

\begin{abstract}
We have studied the dependence of the THG bandwidth on various laser and material parameters. It is found that the bandwidth of a THG signal is strongly dependent on the relative distance of laser focal plane to the material interfaces and laser numerical apertures. The results are important to obtain a high-yield and broad bandwidth THG signal for applications such as characterizations of ultrashort optical pulses.
\end{abstract}

\section{INTROḊUCTION}

Third-harmonic generation (THG) has now been an important tool for applications such as THG microscopy and short-pulse characterization. Unlike second-order processes such as second-harmonic generation, which vanish in isotropic media, THG can be obtained in practically all kinds of material as long as the materials are transparent at the wavelengths involved. In order to enhance yield and resolution (e.g. in microscopy), ultrashort pulses with tightly focused laser beams are often used in THG applications. However, the generation of THG with such strongly focused beams is a complicated issue. For example, in bulk materials, the harmonic signal produced before the focus is reabsorbed coherently behind the focus as a result of Gouy phase shift. In addition, it is well known that, sample interface provides the necessary phase-matching for the THG process. On the other hand, the advances in techniques for generating ultrashort pulses (e.g. $<5 \mathrm{fs}$ ) have raised another important issue about the bandwidth limitations of THG processes. Here we present a detailed bandwidth analysis of THG in optical thin films. Here we use the method of Ward and New [1] for modeling THG with a focused laser beam. The details of the theory can be found in Ref. 1 .

We first study the dependence of the THG yield on relative position of focal plane to the thin film interface. Here we let $F$ be the distance from the focal plane (seen from the incident medium) to the first interface of the thin films. A negative value of $F$ means the focal point is on the left side of the first interface. It is found that the THG yield depends strongly on detuning parameter $\Delta k=3 k_{f}-k_{t h}$, here $k_{f}$ and $k_{t h}$ are the wave vectors of fundamental and TH beams, respectively. In summary, the THG signal has peaks at interfaces of films when the detuning is negative [Fig. 1(a)]. However, when the detuning is positive, the THG signal has a central peaks within the film [Fig. 1(b)]. We also used the theory to study the bandwidth dependence of the THG yield. In figure 1, we show the dependence of THG bandwidth $\Delta k_{\text {FWHM }}$ on the position of focal plane. It is found that a broader THG bandwidth is also obtained at the sample interfaces, [Fig. 1(c)] in addition to a larger THG yield. The results may help to find the optimal position for performing THG experiment with ultrashort pulses, in which larger bandwidth is required. We shall leave other details in the oral presentation.
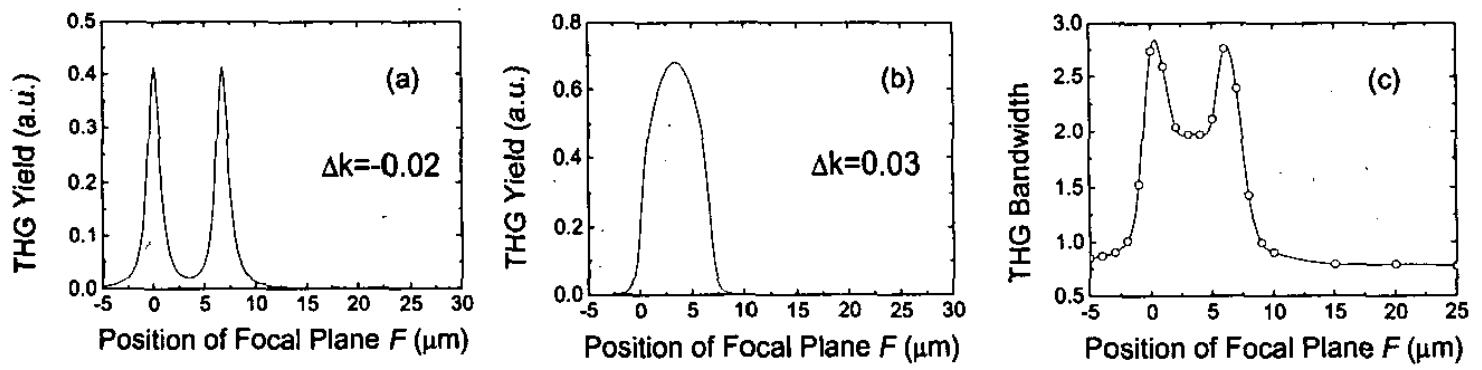

Fig. 1. Dependence of THG yield on focal plane for (a) negative and (b) positive detunings. (c) THG bandwidth dependence on the focal plane.

[1] J. F. Ward and G. H. C. New, "Optical third harmonic generation in gases by a focused laser beam," Phys. Rev., 185, pp. 57 (1969). 